\title{
Information needs of women with breast cancer: A review of the literature
}

\author{
Abbas Sheikhtaheri1(iD), Azin Nahvijou ${ }^{2,3(D)}$, Esmat Mashoof $4^{*}$ (iD) \\ ${ }^{1}$ Department of Health Information Management, School of Health Management and Information Sciences, Iran University of Medical \\ Sciences, Tehran, Iran \\ ${ }^{2}$ Cancer Research Centre, Cancer Institute, Tehran University of Medical Sciences, Tehran, Iran \\ ${ }^{3}$ Research Centre for Breast Diseases, Tehran University of Medical Sciences, Tehran, Iran \\ ${ }^{4} \mathrm{MSc}$ in Health Information Technology, Department of Health Information Management, School of Health Management and Information \\ Sciences, Iran University of Medical Sciences, Tehran, Iran
}

\begin{tabular}{l}
\hline Article Info \\
\hline $\begin{array}{l}\text { Article type: } \\
\text { Review }\end{array}$ \\
\hline Article History: \\
Received: $2020-01-30$ \\
Accepted: $2020-03-08$ \\
Published: $2020-03-25$ \\
* Corresponding author: \\
Esmat Mashoof \\
MSc in Health Information \\
Technology, Department of Health \\
Information Management, School of \\
Health Management and \\
Information Sciences, Iran University \\
of Medical Sciences, Tehran, Iran
\end{tabular}

Email: esmatmashoof@yahoo.com

\begin{abstract}
A B S T R A C T
Introduction: Breast cancer is one of the most common cancers and a serious concern for women's health. Providing sufficient information to these patients increases the level of their participation and improves the quality of their care. Therefore, given the high survival rate of this cancer, it is necessary to understand their information needs. The purpose of this study was to evaluate the information needs of women with breast cancer.
\end{abstract}

Material and Methods: The study is a systematic review of the literature. A search of the databases of PubMed, Scopus, Science Direct and ProQuest has been conducted on studies published in English over the period 20102017. 2881 articles were retrieved and evaluated for title, abstract and full text and after eliminating duplicate and unrelated cases, 18 articles related to the purpose of the study were selected. The articles were then analyzed using content analysis.

Results: Of the 2881 retrieved articles, 18 studies on the information needs of patients with breast cancer were finally reviewed. According to these studies, most information needs were in the areas of diagnosis and treatment (first rank), daily activities (second rank), disease acceptance and self-image (third rank), personal and family life (fourth rank) and sexual health (fifth rank). The most important information needs in the field of diagnosis and treatment was outcomes and side effects of treatment, in the area of daily activities on the impact of disease on social activity, in the area of disease acceptance and self-image was breast reconstruction, body appearance and need for consultation, in the area of personal life, cancer risk for the family and in the area of sexual health was the effect of cancer on sexual attraction were the most cited needs.

Conclusion: Providing information to patients is one of the most important factors in supporting cancer care and understanding the information needs is the first step in seeking information. Patients with breast cancer are interested in receiving information that will help them understand cancer, make decisions about it, and manage their treatment.

Cite this paper as:

Sheikhtaheri A, Nahvijou A, Mashoof E. Information Needs of Women with Breast Cancer: A Review of the Literature. Front Health Inform. 2020; 9: 30. DOI: 10.30699/fhi.v9i1.216

\section{INTRODUCTION}

Breast cancer is the deadliest form of malignancy among women [1] and is a serious concern for women's health [2]. According to statistics from the International Agency for Research on Cancer, in 2012 there was a sharp increase in breast cancer [ $\underline{3}]$. The disease is more prevalent in developed and high-income countries due to the higher prevalence of risk factors, such as old age at first pregnancy, high calorie intake, and the use of alternative hormone therapies [4]. Prolonged illness or even physical consequences, psychological symptoms and deficiencies can diminish the quality of life. In fact, these patients may experience painful symptoms and complications for several years after diagnosis. Therefore, efforts to improve physical function and improve quality of life in these patients are a necessity [ㅁ] .

According to the evidence, patients with breast 
cancer suffer from physical, mental and social problems that persist long after initial treatment. They therefore have a serious need for training, information and support over time []]. On the other hand, women are an important foundation in any family and society, therefore, improving the quality of life of women with breast cancer not only improves their survival but also improves their family structure []ㅡ. Therefore, efforts to empower these patients to raise their awareness, skills, and motivation to positively impact their health are needed to manage cancer-related problems, especially breast cancer, and to meet the information needs and improve the quality of life of these patients []].

Empowerment by building confidence and providing information to patients to take care of themselves improves population health and encourages healthy behaviors and helps prevent long-term illness []. According to studies, patients are interested in receiving information to help them understand cancer, make decisions about it, and manage their treatment, and patients' satisfaction with the information needs is related to perceived quality of care, quality of life, mental health, and health improvement. []]. Researchers concluded that appropriate information is a prerequisite for informed decision making [10]. According to Husson et al., providing information to patients is one of the important factors in support of cancer care and leads to increased ability to cope with the disease and improve, prepare patients for treatment, and increase adherence to treatment [11]. Other researchers argued that all patients should have access to sufficient information to plan and respond to their problems and to change them [12].

On the other hand, results of systematic review studies show that 6 to $93 \%$ of cancer patients reported providing sufficient information as an unmet need [11]. Survivors have also been shown to have poor health-related quality of life 2 to 5 years after diagnosis with high levels of unmet needs. There has also been evidence of increased depression and anger caused by the disease [13].

To address these challenges, it is necessary to provide awareness, knowledge, skills and motivation to make a positive impact on patients' health status [14]. In addition, providing patients with relevant information and tailored to their needs can help them understand the expected results and deal with unexpected outcomes [12]. On the other hand, understanding information needs is the first step in seeking information [15].

Patients with breast cancer have a great deal of information needs [16]. According to studies, the information provided to them eliminates their anxiety and helps them make decisions. The amount and type of information a person receives varies according to the patient's information needs based on the disease, its stage, the patient's age, their culture and beliefs. If patients are more informed and involved in decision making, the results of patient care will be better [17]. Various studies have been conducted around the world to address the information needs of patients with breast cancer in different dimensions, including information needs assessment of women over 65 [18], women over 75 [19], women with advanced breast cancer [20], women with chemotherapy-treated breast cancer [21], women with breast cancer surgery [22], women with newly diagnosed breast cancer [23], women with lymphedema [24], women with breast cancer undergoing radiotherapy [25], women with menopause [26], and women with mastectomy [27] which in addition to a number of common needs have reported specific and unique needs. In this study, the goal is to identify the information needs of all these groups, to take a step toward prioritizing, comparing and classifying the information needs and to create conditions for improving the information available to patients.

\section{MATERIAL AND METHODS}

This study is a systematic review in which English articles on information needs of breast cancer patients with keywords informational needs, information needs, breast cancer, breast neoplasm, cancer, patient, from 2010 to 2017 were searched. PubMed, ProQuest, Scopus and Science Direct were searched. The search strategy is shown in Table 1 and the search result is in PRISMA chart (Fig 1).

Table 1: Search strategy

\begin{tabular}{|l|l|}
\hline & $\begin{array}{l}\text { PubMed, Science direct, } \\
\text { Scopus, ProQuest }\end{array}$ \\
\hline A1 & $\begin{array}{l}\text { informational needs } \\
\text { OR information needs }\end{array}$ \\
\hline A2 & $\begin{array}{l}\text { breast cancer } \\
\text { OR breast neoplasm } \\
\text { OR cancer }\end{array}$ \\
\hline A3 & Patient \\
\hline Strategy & (A1 AND A2 AND A3) \\
\hline
\end{tabular}

\section{Inclusion and exclusion criteria}

Inclusion criteria included: Access to the full text of the articles, relevance to the subject, English language and publication in the 2010-2017 timeframe and were performed solely on patients with breast cancer. In this study, all quantitative and quantitative part of mixed methods (quantitativequalitative) studies on patients' information needs were included. Articles that included breast cancer patients in addition to patients with other types of cancer, abstracts of conferences, a letter to the editor, as well as articles exploring the views of non- 
patient groups, articles in a language other than English and articles on men with breast cancer, qualitative studies, and review studies were excluded.

\section{Data extraction and analysis}

Data were extracted and analyzed in four stages. After screening the article titles, the abstracts were reviewed. Then, the full text of the articles was reviewed and the articles were included in the final analysis according to the inclusion and exclusion criteria. Data were extracted by two authors using a data extraction form. The variables studied in each article for data extraction were: title, author, year of publication, method, study population and findings.

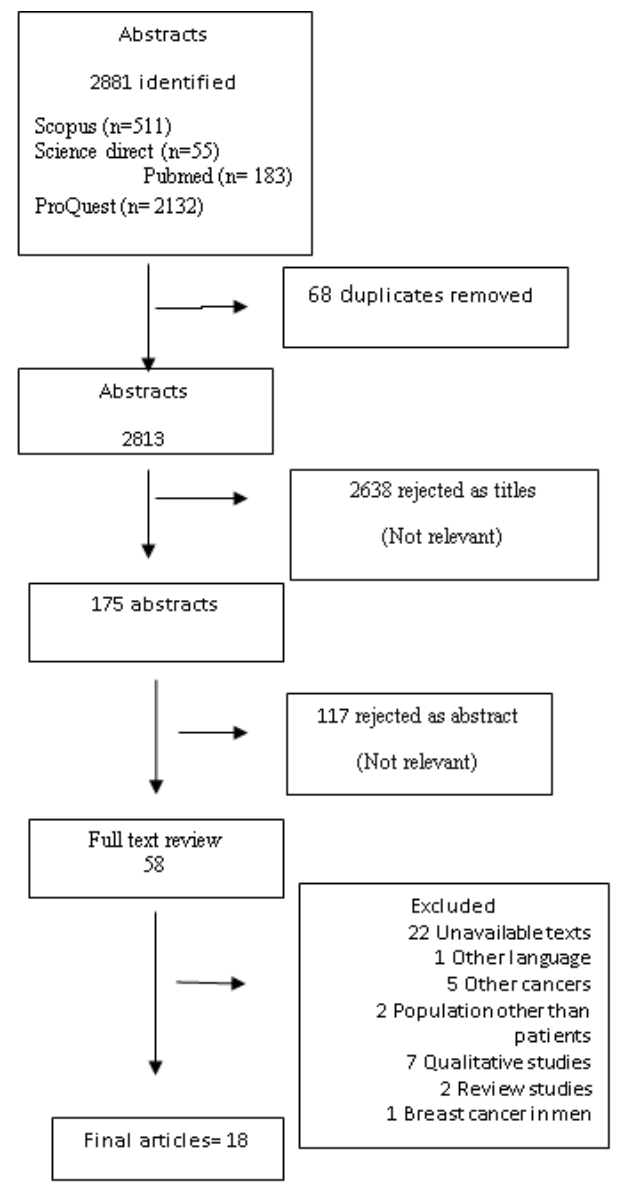

Fig 1: The process of the selection of the relevant studies

\section{RESULTS}

A total of 2881 articles were retrieved from the databases. After eliminating duplicate and irrelevant articles based on title, abstract and full text evaluation, finally 18 articles related to the purpose of the study were selected (Fig 1). Based on the survey, 18 out of the 16 studies were quantitative (cross-sectional descriptive-) and two were mixed methods (quantitative-qualitative).

In terms of countries studied, studies conducted in Asia included 7 studies (38.9\%), European countries
6 studies (33.3\%), American continents 3 studies $(16.7 \%)$ and Australia 2 studies (9.5\%). In terms of the study publication year, there has been a steady trend between 2011 and 2012. It declined in 2013 and then increased between 2013 and 2015 and again slightly decreased in 2016 (Fig 2).

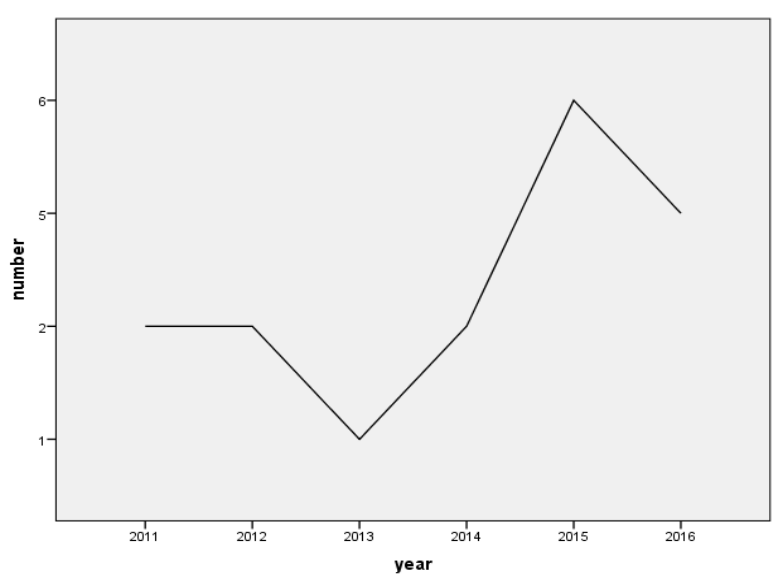

Fig 2: Publication trends by years

The full results of the literature review are presented in Table 2, classification of information needs; Table 3, information needs of women with breast cancer (Quantitative Studies) and Table 4, Information needs of women with breast cancer (Quantitative-Qualitative Studies).

According to studies, information needs were categorized into six general categories and several subcategories (Table 2). Diagnosis and treatment (18 studies), comprising the largest percentage of studies, and then daily activities were ranked second (10 Studies), disease acceptance and self- image ranked third (9 studies), personal and family life ranked fourth (7 studies) and sexual health ranked fifth (5 studies). Seven studies were categorized in the "other needs" group.

In the field of diagnosis and treatment, the need for information on outcomes and side effects has been emphasized in 9 studies (50\%), which constitutes the largest number of studies. According to studies, the most frequently reported information needs of patients after adverse events, were treatment options, probability of recovery and treatment, progression of disease and treatment plan. Other needs are of lesser importance to patients, but as an information needs have been requested from patients (Table 2). In the domain of daily activities, the need for information on the impact of the disease on social activity and daily activity in seven studies (38.9\%) and self-care in four studies $(22.2 \%)$, in the area of disease acceptance and selfimage, the need for information in the field possibility of breast reconstruction, body appearance, prosthesis and need for counseling and 
phobia counseling in each of the five studies (27.8\%) were most common. In the area of personal and family life, requiring information on cancer risk for the family in four studies $(22.2 \%)$ and in the area of sexual health, the need for information on the impact of sexual attraction and emotion was emphasized in three studies (16.7\%). These were the most frequently requested information reported in the studies.

\section{Table 2: Classification of information needs by six general categories}

\begin{tabular}{|c|c|c|}
\hline $\begin{array}{l}\text { The main classes of } \\
\text { information needs }\end{array}$ & Subcategories & Frequency \\
\hline $\begin{array}{l}\text { Diagnosis and } \\
\text { treatment }\end{array}$ & $\begin{array}{l}\text { Side effects of treatment ( } 9 \text { studies), Treatment options (6 studies), Likelihood of cure/ } \\
\text { treatment ( } 5 \text { studies), Progress of disease, Treatment plan (Each one has } 4 \text { studies), } \\
\text { Disease management, Managing lymphedema, Hereditary in breast cancer, Health } \\
\text { promotion (Each one has } 3 \text { studies), Follow-up after treatment, Stages of disease, } \\
\text { Nutrition during treatment, Skin care, Drug information, The effects of treatment on the } \\
\text { body, Symptoms(Each one has } 2 \text { studies), Medical examination results, Emotions related } \\
\text { to the situation, Measures of rehabilitation, Benefits of treatment, Quality of life, Clinical } \\
\text { test, Diagnosis, The best approach to treatment, Risk of metastasis or recurrence, The best } \\
\text { treatment, Prevention of postoperative problems, Fatigue management, Severe disability, } \\
\text { Causes, Duration of the treatment, How to be ready for treatment, The reason for } \\
\text { selected treatment, Why there are cameras and computers in the treatment room and what } \\
\text { they are used for, The reason for radiation therapy, Symptoms and prevention of } \\
\text { postoperative problems, Description of the treatment, X-rays importance for the treatment, } \\
\text { What will happen after treatment, Complementary treatment, Signs and symptoms of } \\
\text { lymphedema, Port catheter, Possible effects of the disease, Genetic counseling, Being a } \\
\text { carrier, Risk of breast cancer, Lymphedema, Importance of lymphedema, What happens } \\
\text { on the first day, Adverse effect of treatment, Help and support at home, Breast } \\
\text { examination, Postoperative hospital and home care practices, Rehabilitation services, How } \\
\text { effective the treatment has been for other patients (Each one has one studies) }\end{array}$ & 18 studies \\
\hline Daily activity & $\begin{array}{l}\text { Effects of breast cancer on occupational life, daily activity ( } 7 \text { studies), Home self-care (4 } \\
\text { studies), Shoulder and arm exercises, Exercise and sport ( } 3 \text { studies), Healthy Life style } \\
\text { (one study) }\end{array}$ & 10 studies \\
\hline $\begin{array}{l}\text { Disease acceptance } \\
\text { and self- image }\end{array}$ & $\begin{array}{l}\text { Breast reconstruction, Body image and appearance, Prosthesis, Psychological care during } \\
\text { treatment, Coping with fears ( } 5 \text { studies), } \\
\text { Presence of support groups / Available consultations (4 studies), Physical/ psychosocial } \\
\text { services, Physical/ psychosocial issues ( } 2 \text { studies) }\end{array}$ & 9 studies \\
\hline $\begin{array}{l}\text { Personal and family } \\
\text { life }\end{array}$ & $\begin{array}{l}\text { Risk of breast cancer for sisters or daughters (4 studies), Effect of disease on family } \\
\text { and friends ( } 3 \text { studies), Breastfeeding capacity, Fertility ( } 2 \text { studies), Effect of treatment } \\
\text { on usual social activities or personal life, Contraception (one study) }\end{array}$ & 6 studies \\
\hline Sexual health & $\begin{array}{l}\text { The effect of breast cancer on body and sexual attractiveness ( } 2 \text { studies), The effect of } \\
\text { breast cancer on sexual activities, Sexual outcome of the disease, Information for partner, } \\
\text { Difficulties in being aroused, Pain during intercourse, Written information on sexual } \\
\text { alteration, Difficulties in reaching orgasm, Anxiety about sex, Sexual well-being across } \\
\text { stages of care, Sex aids and products, Irregular menstruation, Sexual well-being for } \\
\text { couples, Partner satisfaction, Increased sensitivity, Physical changes, Loss of sensation, } \\
\text { Relationship communication, How to talk about sex and intimacy (one study) }\end{array}$ & 5 studies \\
\hline Other & $\begin{array}{l}\text { The cost of treatment ( } 2 \text { studies), Insurance, working age, Better therapeutic center, Being } \\
\text { reinsured about the future, Financial problems, The name of radiation oncologist, The } \\
\text { roles of the different staff in the department, Who can provide me with information, } \\
\text { Parking, Contact person at the time of lymphedema, Physical burden, Relationship } \\
\text { changes, Where to go for support, How to talk to health professionals, Loss of identity, } \\
\text { Appointment, Vacations and travel (one study) }\end{array}$ & 7 studies \\
\hline
\end{tabular}


Table 3: Information needs of women with breast cancer (Quantitative studies)

\begin{tabular}{|c|c|c|c|c|}
\hline 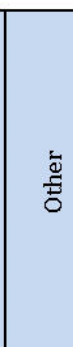 & 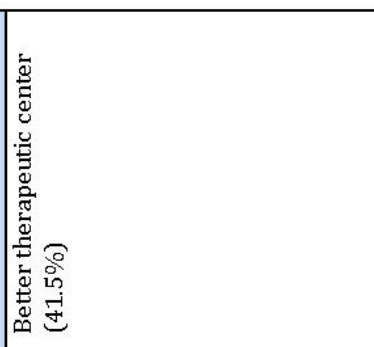 & & 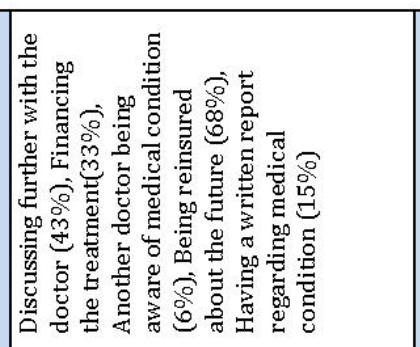 & \\
\hline 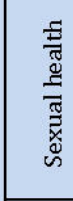 & 1 & 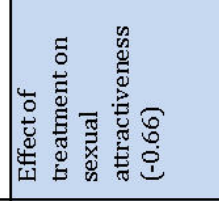 & 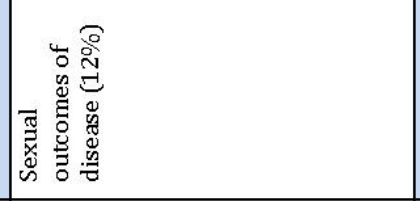 & \\
\hline 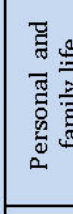 & 1 & 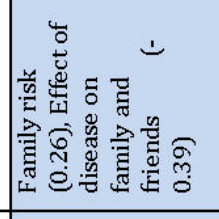 & 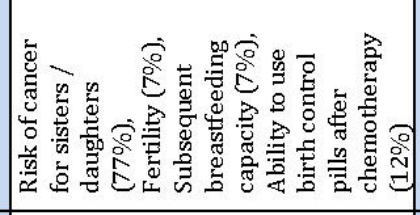 & \\
\hline 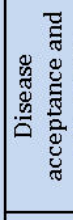 & 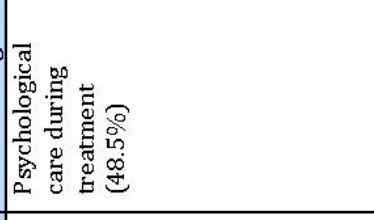 & 1 & 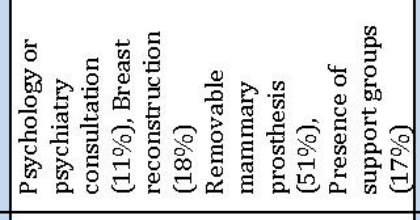 & \\
\hline 忿育 & & 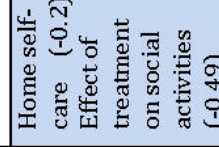 & 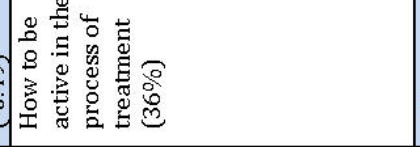 & 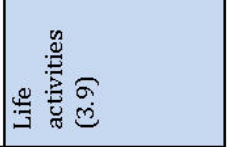 \\
\hline 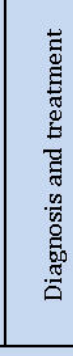 & 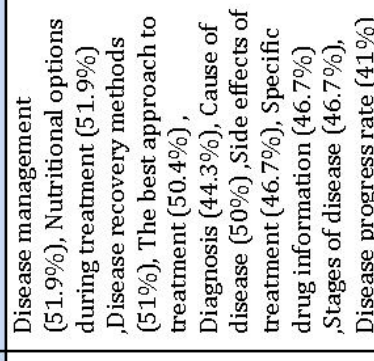 & 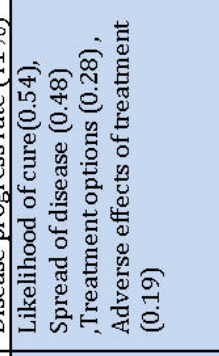 & 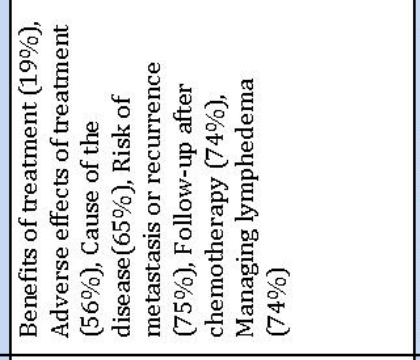 & 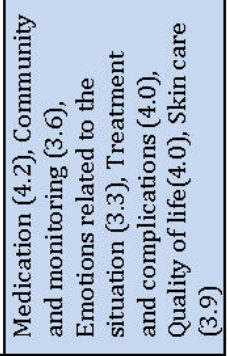 \\
\hline 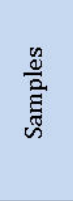 & 密 & 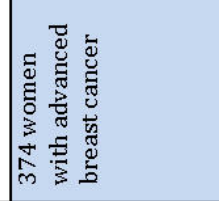 & 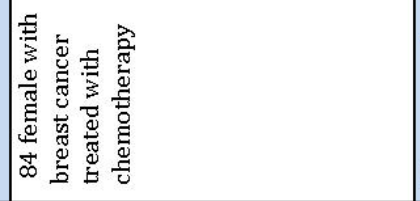 & 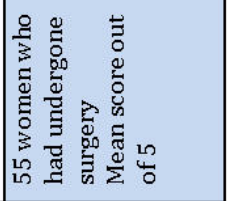 \\
\hline $\begin{array}{l}\text { 总 } \\
\frac{d}{2}\end{array}$ & 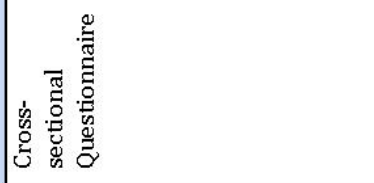 & 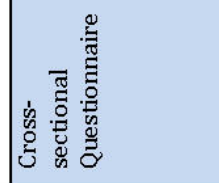 & 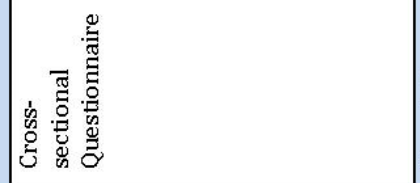 & 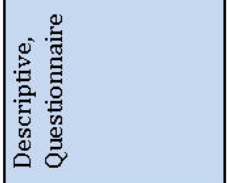 \\
\hline 产 & $\stackrel{\Xi}{\Xi}$ & 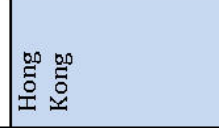 & 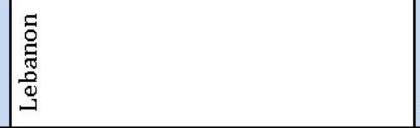 & 尊 \\
\hline 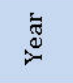 & 品 & 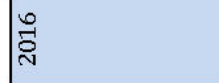 & 产 & 菋 \\
\hline 总 & s: & 晜 & 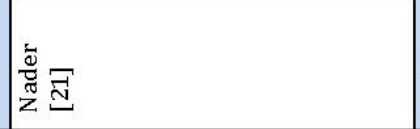 & 暗 \\
\hline
\end{tabular}


Table 3: Continued

\begin{tabular}{|c|c|c|c|c|}
\hline 离 & 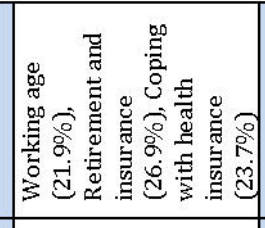 & & 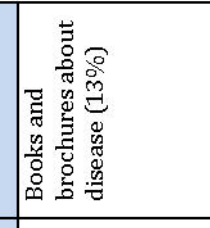 & 1 \\
\hline 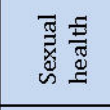 & 1 & & & \\
\hline & & & & \\
\hline 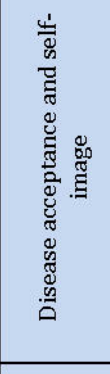 & 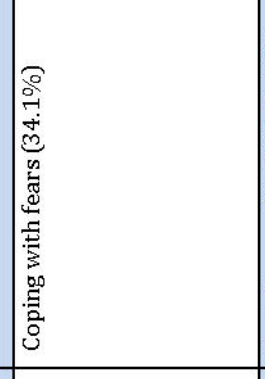 & 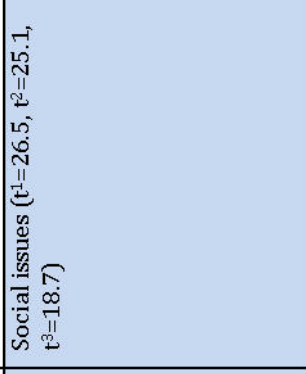 & 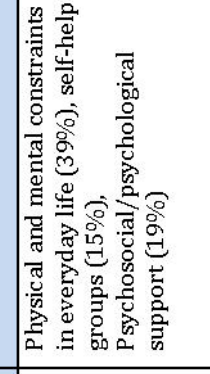 & 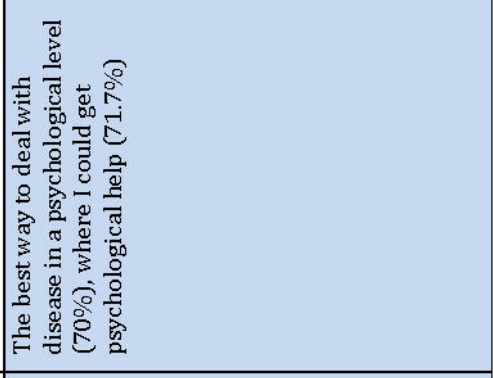 \\
\hline 章蛋 & & & 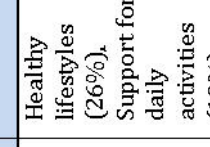 & 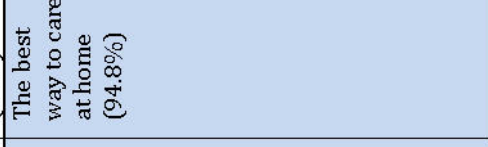 \\
\hline 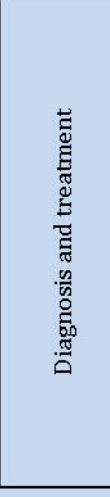 & 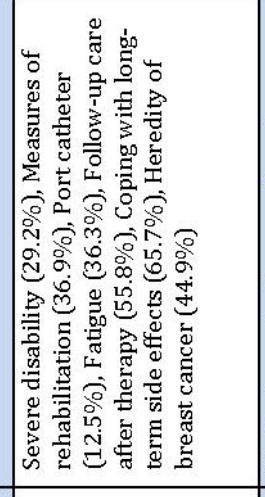 & 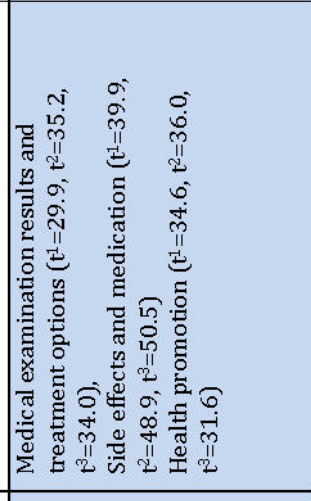 & 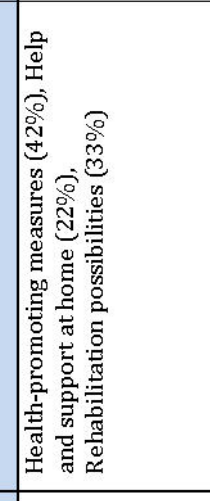 & 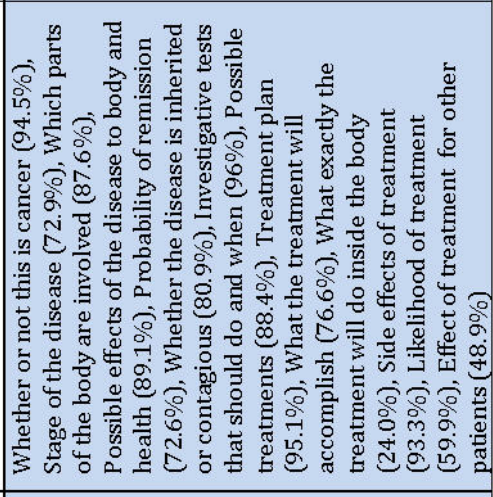 \\
\hline 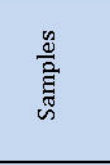 & 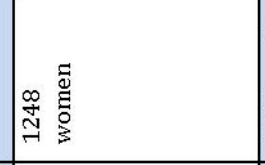 & 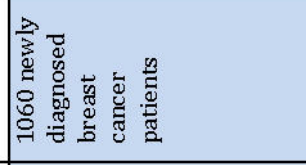 & 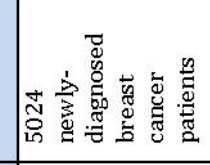 & 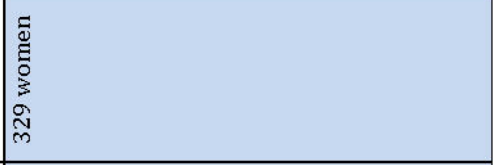 \\
\hline 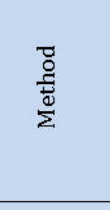 & 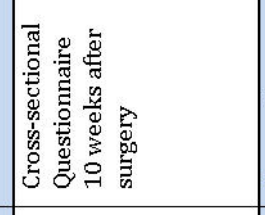 & 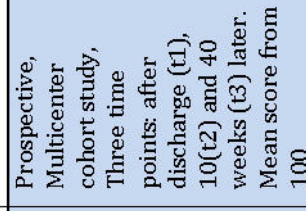 & 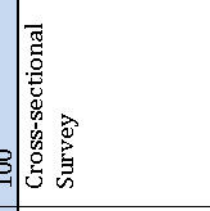 & 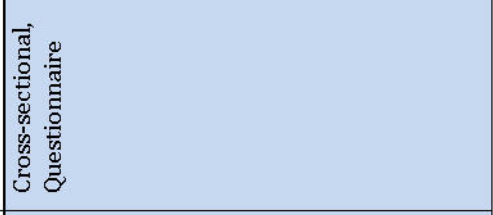 \\
\hline 量 & 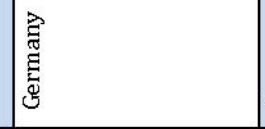 & 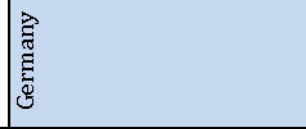 & 窟 & : \\
\hline 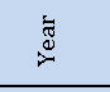 & 总 & 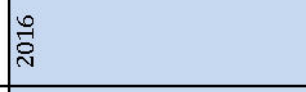 & 嘀 & ָे \\
\hline 亳 & 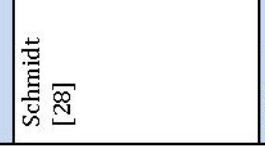 & 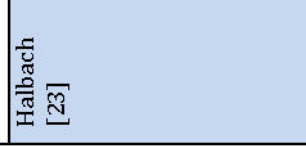 & 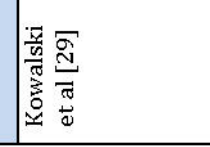 & 列 \\
\hline
\end{tabular}


Table 3: Continued

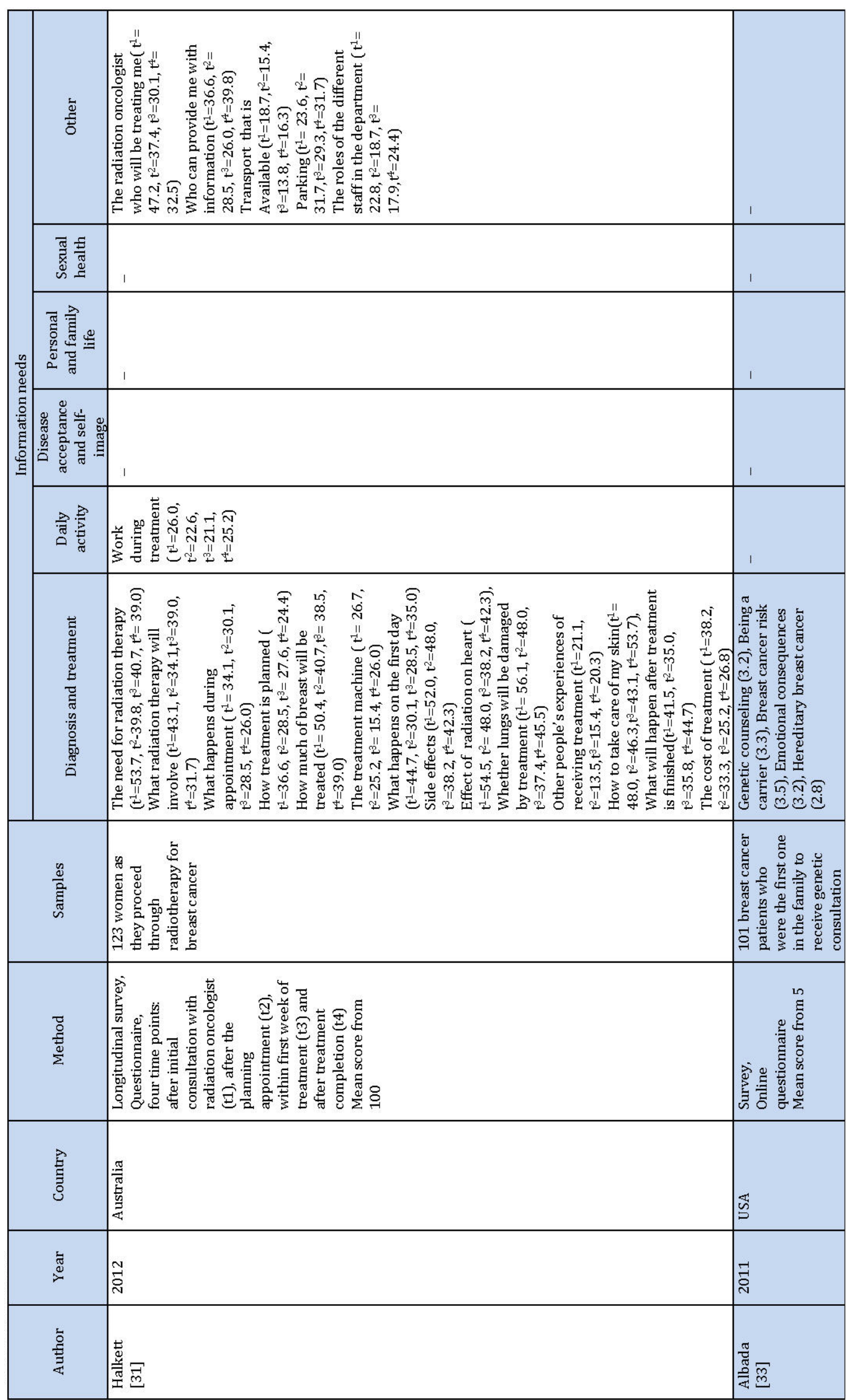


Table 3: Continued

\begin{tabular}{|c|c|c|c|c|}
\hline 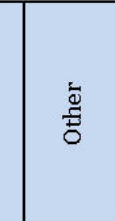 & 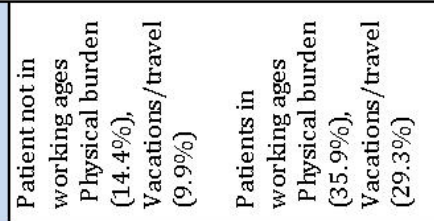 & 1 & 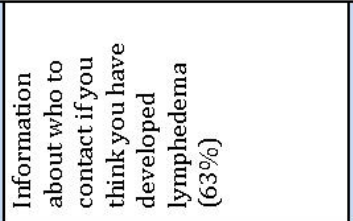 & , \\
\hline 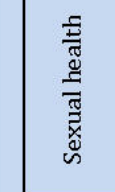 & । & 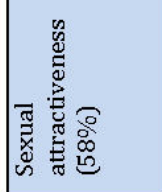 & , & । \\
\hline 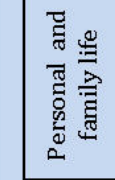 & 1 & 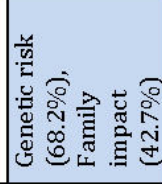 & & 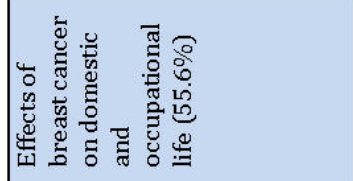 \\
\hline 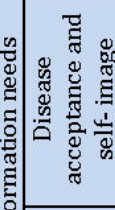 & . & 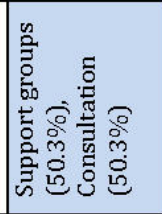 & 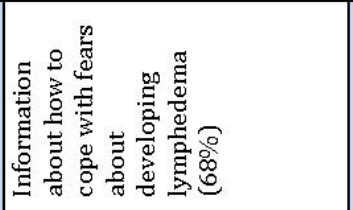 & 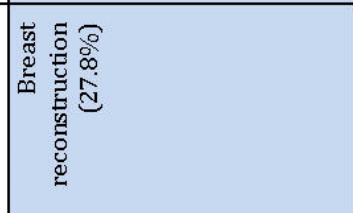 \\
\hline 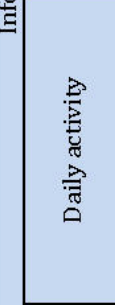 & 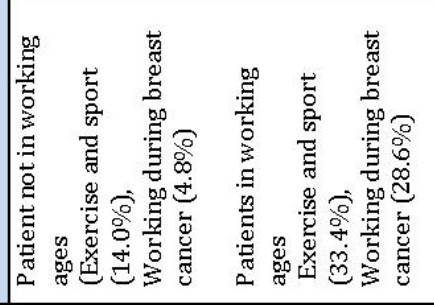 & 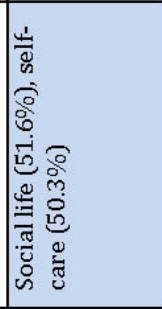 & & 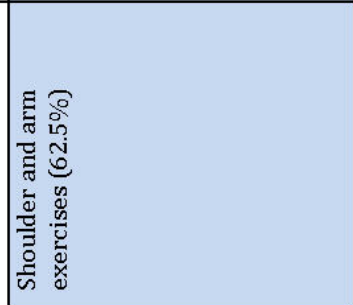 \\
\hline 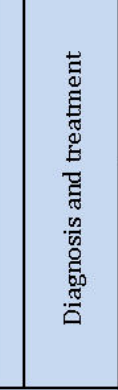 & 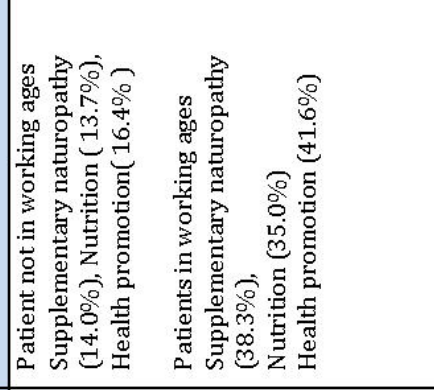 & 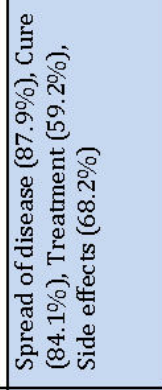 & 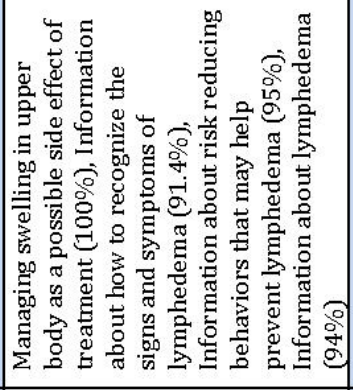 & 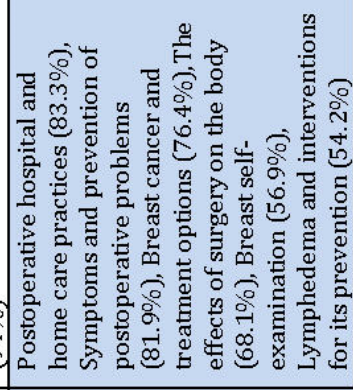 \\
\hline $\begin{array}{l}\frac{n}{0} \\
\frac{0}{\tilde{n}} \\
\tilde{n}\end{array}$ & 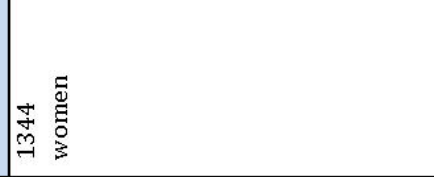 & 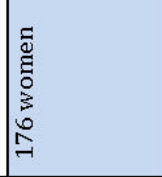 & 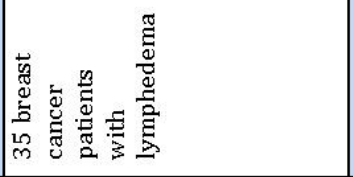 & 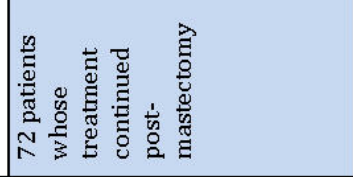 \\
\hline 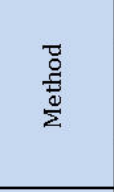 & 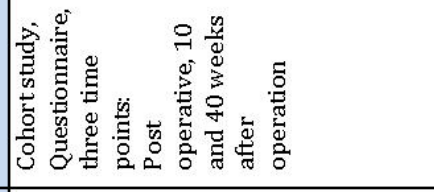 & 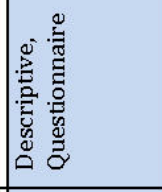 & 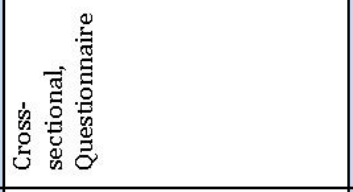 & 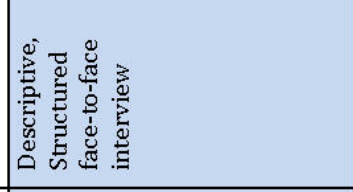 \\
\hline 蔦 & 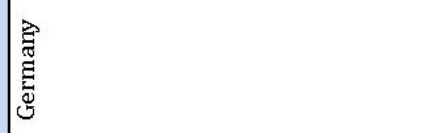 & 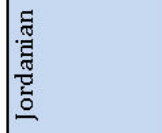 & 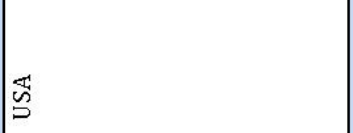 & 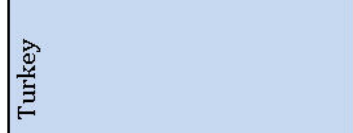 \\
\hline$\stackrel{\bar{\varpi}}{\check{\varpi}}$ & 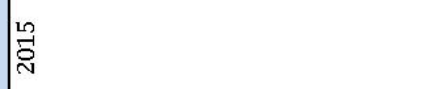 & ت্ं & 空 & 号 \\
\hline 童 & 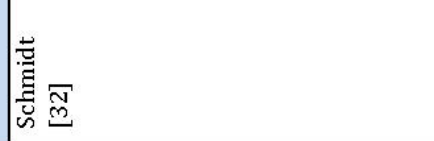 & 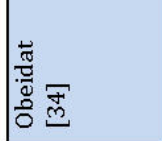 & 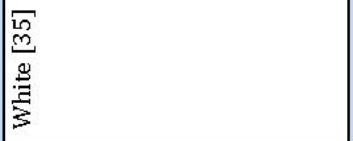 & 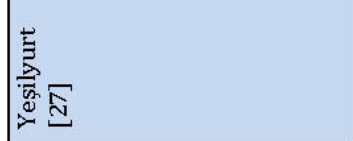 \\
\hline
\end{tabular}


Table 3: Continued

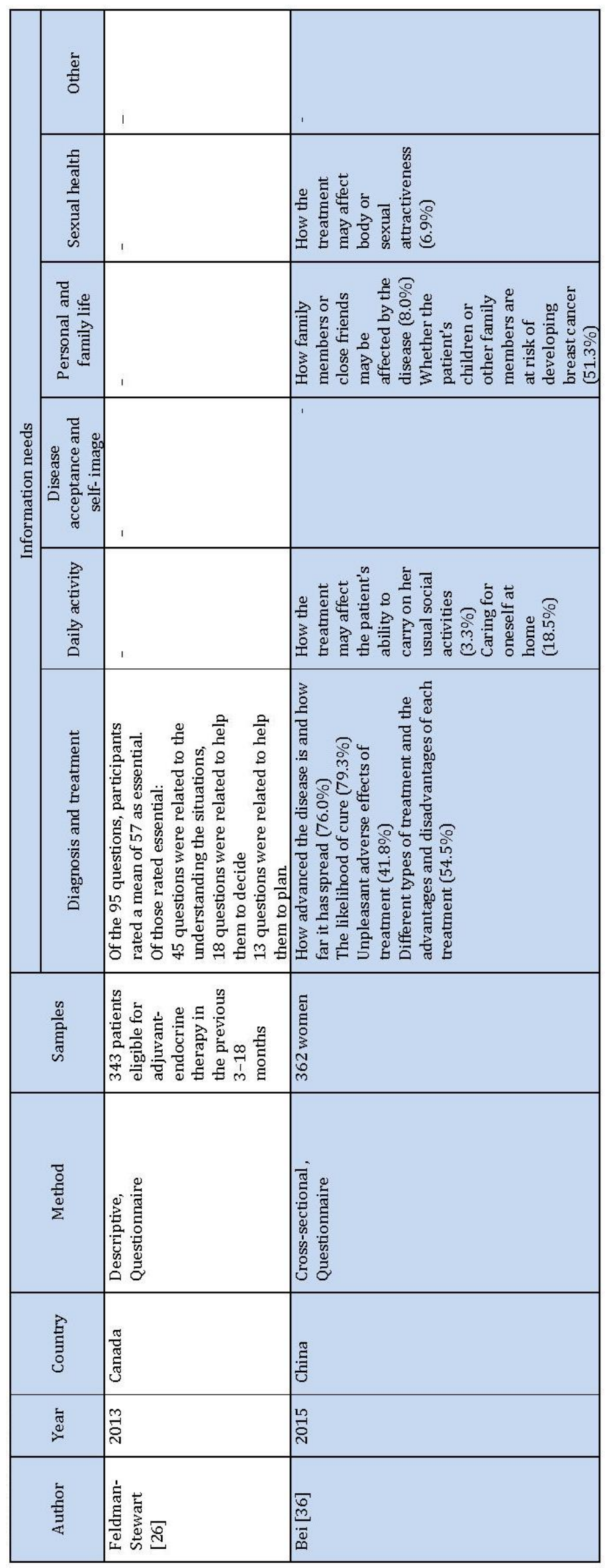


Table 4: Information needs of women with breast cancer (Mixed method studies)

\begin{tabular}{|c|c|c|}
\hline 离 & 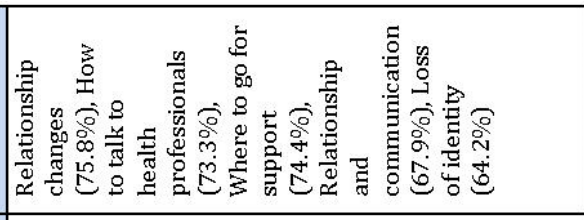 & , \\
\hline 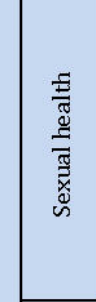 & 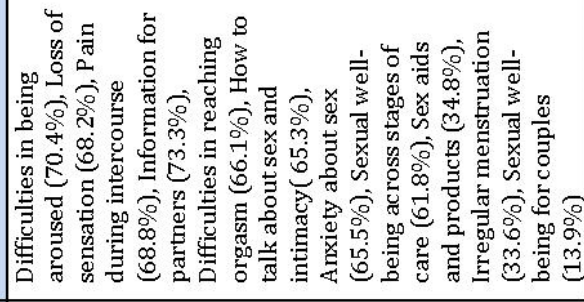 & \\
\hline 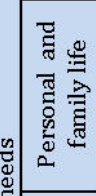 & 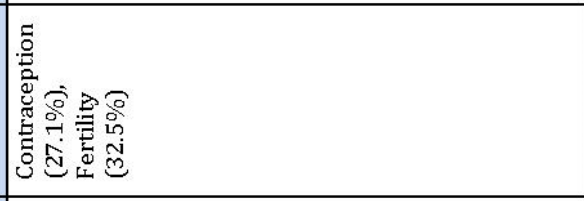 & I \\
\hline 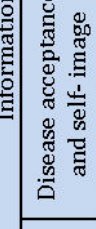 & 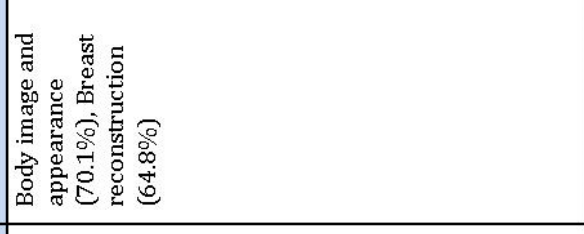 & 1 \\
\hline 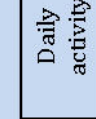 & & \\
\hline 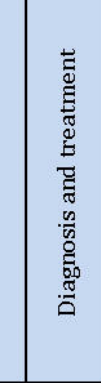 & 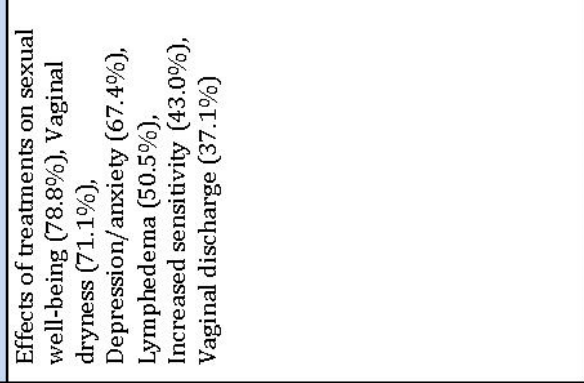 & 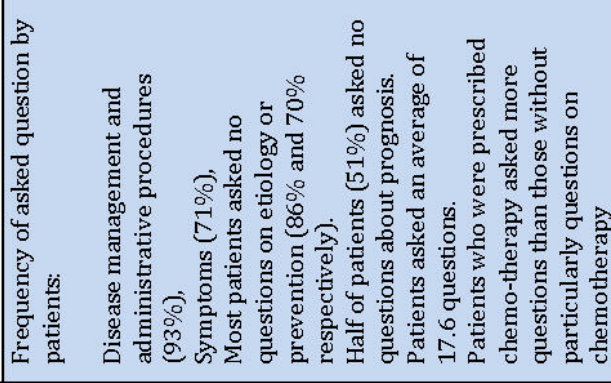 \\
\hline 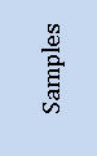 & 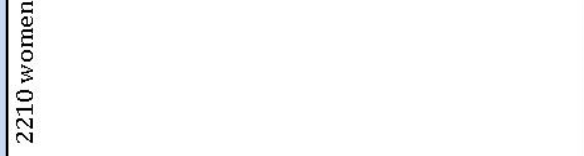 & 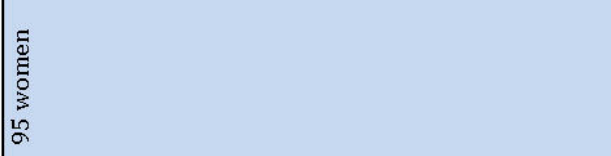 \\
\hline 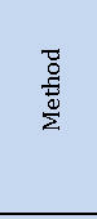 & 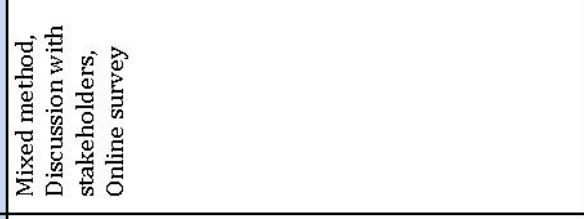 & 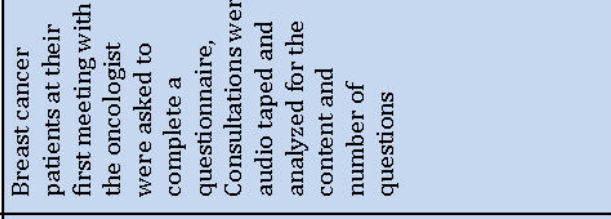 \\
\hline 㝘 & $\mid$ & $\frac{\mathrm{z}}{\underline{\underline{Q}}}$ \\
\hline 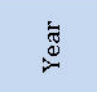 & ज̃ & 商 \\
\hline 竧 & 亮 & 商然 \\
\hline
\end{tabular}




\section{DISCUSSION}

Information needs arise because of dissatisfaction with the status quo or a defect in the knowledge of the individual. The information needs of breast cancer patients are related to different factors. The present study showed that the trend of studies on the information needs of patients with breast cancer was declining and relatively constant between 2011 and 2013 and then increasing between 2013 and 2015 and slightly decreased in 2016. According to the present study, most information needs of women with breast cancer were in the field of diagnosis and treatment, which was mentioned in $100 \%$ of the studies. In this area, information about side effects (50\%), treatment options $(33.3 \%)$ and the probability of recovery or treatment of the disease $(27.8 \%)$ was higher than the others.

The present study showed that women with breast cancer, with any culture and language, with any age group or country of residence in terms of level of development, prioritize their information needs in the field of diagnosis and treatment. These patients, regardless of cancer progression (metastatic or nonmetastatic), treatment stage (initiation or end of treatment), type of treatment (whether radiotherapy or chemotherapy or surgery) or occupation (whether they are employed or housewives), information about diagnosis and treatment is their most important information need, indicating the importance of this area and its subcategories among these patients. Previous studies have also addressed this issue [10, 19, 2640].

Other important results in this study are the importance of familiarizing with the side effects of treatment and medication, its management and control and familiarity with different treatment options. Most newly diagnosed patients, either those who have received the first course of treatment and those whose cancer has progressed or are undergoing radiotherapy, are interested in having information about side effects of the disease, treatment, medication, and treatment options [ $[\underline{18}$, 40-47].

According to the present study, information needs in the area of daily activities were ranked second. The most important sub-categories in this area are the impact of the disease on social activity, daily activity and self-care, and occupation during the illness was a concern for employed women. Previous studies have also addressed this issue [48-50]. Interruptions during recovery can increase the burden of the disease and respond to this need is very important in initiating treatment [32]. Among the different patient groups, literate and employed women and survivors at the age of menopause were among the groups whose information needs on complementary therapies was important $[\underline{32}, \underline{51}]$. In the Miyashita study, only $25.2 \%$ of participants received information about dietary supplements and alternative diets [52]. Based on previous studies, information sources for alternative and complementary therapies are outside the medical system [53] and participants preferred not to receive this information from medical professionals, and one quarter of study participants reported unmet needs on alternative and complementary therapies [54]. Patients receive complementary and alternative therapies on the recommendation of family and friends without sufficient information about complementary therapies [ㄷ5]. Therefore, providing evidence-based counseling on complementary alternatives to meet the patients' needs and designing guidelines to assist and clarify evidence is essential [ $\underline{56}$.

Self-care education was another priority for patients. Self-care is the behaviors that individuals use to manage and improve their health [57]. Selfcare education has a long-term impact on people's health status [ [58]. Chiaranai showed that better selfcare leads to better quality of life [59]. Williams also showed in his study that self-care assessment can be learned and has a positive effect on the management of adverse events and the patients' participation in effective self-care [60].

Another result of this study was the need for information on the possibility of breast reconstruction, body appearance and the need for consultation in this area. Previous studies have also shown that women have a high level of body image problems and emotional responsibilities [61]. Symptoms of traumatic stress are more frequent in young women with breast cancer, those receiving post-operative treatment, and those with low selfefficacy [62]. Discomfort with the diagnosis and treatment of breast cancer disrupts women's emotional well-being, family and work life and reduces their quality of life [63]. They avoid social environments because of concerns about questions and people's curiosity. Social support plays a major role in helping women with breast cancer [64] and if they have adequate social support, disease management will be easier for them [65]. The support they receive from nurses during the diagnosis and treatment of illness is also important [64].

According to the present study, breast cancer survivors are concerned about the impact of the disease on sexual feelings and attractiveness, decreased sexual activity and sexual outcomes resulting from the disease, and are also keen to have information on identifying support sites and how to talk to specialists $[\underline{20}, \underline{21}, \underline{34}, \underline{37}, \underline{44}, \underline{66}]$. The result was the opposite of Lei's study, which showed that information about sexual attraction and body image 
was not of concern to patients at the Malaya University Center [10]. The impact of sexual changes lasts long after successful treatment and is often accompanied by physical and emotional side effects [67]. Studies have shown that women with breast cancer have lower levels of sexual satisfaction than healthy women [68]. According to research, the strongest predictor of consistent sexual problems after breast cancer is low sexual attractiveness [69]. Women who have poor body image after breast cancer have lower sexual satisfaction and are more dissatisfied with their sex [68]. While sexual intimacy makes cancer experiences more manageable and contributes to the recovery process [궁, and it serves as an important factor for spouse closeness and quality of life in palliative care [71]. In their study, Stabile found that $87 \%$ of patients with breast and genital cancers considered the role of sexual health important in quality of life, but most reported lack of information in this regard [72]. Li found in his study that sexual health information needs were lower in priority than diagnosis, disease spread, and improvement [20] with one exception, in which it was reported that sexual attraction for newly diagnosed Malaysian women with breast cancer was reported as the second most important information needs [국. Also, in Takahashi's study, information on treatment-related sexual changes was the most important sexual information needs for Japanese women [74]. In Gould's study, young women with breast cancer showed significant unanswered information needs related to premature menopause and sexual dysfunction [75]. Ussher's study showed that a wide range of sexual health information is needed to meet the specific needs of people with breast cancer, and $78 \%$ of patients found it important to have information about the impact of treatment on sexual health [37]. In one Australian study, only $30 \%$ of couples received sex information, the majority of whom were dissatisfied [75] and in the Ussher's study, two-thirds did not receive the information they needed [37].

Nurses and health professionals can play a key and important role in addressing concerns about sex after breast cancer [76] but most of them not talk about sexual intercourse because of a lack of knowledge or experience or not accepting it as one of their duties. Therefore, it seems necessary to reinforce the problem of education by professionals [37].

One of the strengths of this study was to investigate the information needs of women with breast cancer in different age, cultural, social, and therapeutic situations. In future studies, it is suggested to examine the information needs of the population of men with breast cancer.

\section{CONCLUSION}

Having information about diagnosis and treatment is one of the most important information needs of women with breast cancer and on the other hand, it seems that the information provided by physicians is not always responsive to patients' concerns. Therefore, the need for long-term education and support of breast cancer patients seems to be essential. Dedicated clinics and groups for patient education after diagnosis, creating a space for faceto-face question and answer with care providers, free and fast access to complete and accurate information, creating, organizing, managing and updating dedicated web pages and Internet web sites related to topics and counseling services to patients and their families on disease, treatment, nutrition, complications, and depression management can help patients obtain adequate and appropriate information and meet their information needs.

\section{ACKNOWLEDGEMENT}

This article is part of the Master thesis supported by Iran University of medical sciences (IUMS/SHMIS 95/9311304010). The research ethics committee of Iran University of medical science confirmed the proposal of this study (IR.IUMS.REC 1395.9311304010).

\section{AUTHOR'S CONTRIBUTION}

The authors agree on this final form of the manuscript, and attested that all authors contributed in the final draft of the manuscript.

\section{CONFLICTS OF INTEREST}

The authors declare no conflicts of interest regarding the publication of this study.

\section{FINANCIAL DISCLOSURE}

This project has been conducted by a grant from cancer research center of cancer institute of Iran (Sohrabi cancer charity, Grant No: 37350-202-0197).

\section{REFERENCES}

1. Mousavi SM, Montazeri A, Mohagheghi MA, Jarrahi AM, Harirchi I, Najafi M, et al. Breast cancer in Iran: An epidemiological review. Breast J. 2007; 13(4):
383-91. PMID: 17593043 DOI: 10.1111/j.15244741.2007.00446.x [PubMed]

2. Andic S, Karayurt O. Determination of information and support needs of first degree relatives of women with breast cancer. Asian Pac J Cancer Prev. 2012; 
13(9): 4491-9. PMID: 23167367 DOI: 10.7314/apjcp.2012.13.9.4491 [PubMed]

3. Breast cancer [Internet]. 2017 [cited: 18 Feb 2018]. Available at: http://globocan.iarc.fr/old/FactSheets/cancers/brea st-new.as.

4. Taghavi A, Fazeli Z, Vahedi M, Baghestani AR, Pourhoseingholi A, Barzegar F, et al. Increased trend of breast cancer mortality in Iran. Asian Pac J Cancer Prev. 2012; 13(1): 367-70. PMID: $22502702 \quad$ DOI: $10.7314 /$ apjcp.2012.13.1.367 [PubMed]

5. Shahsavari H, Matory P, Zare Z, Taleghani F, Kaji MA, et al. Effect of self-care education on the quality of life in patients with breast cancer. J Educ Health Promot. 2015; 4:70. PMID: 27462612 DOI: 10.4103/22779531.171782 [PubMed]

6. Hong-li C, Xiao-Chun X, Jiang-Bin J, Jing-Bo Z, Yao W. Quality of life in patients with breast cancer and their rehabilitation needs. Pak J Med Sci. 2014; 30(1): 126-30. PMID: 24639845 DOI: $10.12669 /$ pjms.301.3952 [PubMed]

7. Kuijpers W, Groen WG, Oldenburg HS, Wouters MW, Aaronson NK, van Harten WH. Development of MijnAVL, an interactive portal to empower breast and lung cancer survivors: An iterative, multistakeholder approach. JMIR Res Protoc. 2015; 4(1): e14. PMID: 25614924 DOI: 10.2196/resprot.3796 [PubMed]

8. Self Care Forum. What do we mean by self care and why is it good for people? [Internet]. 2017 [cited: 1 Jan 2018]. Available at: http://www.selfcareforum.org/about-us/what-dowe-mean-by-self-care-and-why-is-good-for-people.

9. Matsuyama RK, Wilson-Genderson M, Kuhn L, Moghanaki D, Vachhani H, Paasche-Orlow M. Education level, not health literacy, associated with information needs for patients with cancer. Patient Educ Couns. 2011; 85(3): e229-36. PMID: 21543182 DOI: 10.1016/j.pec.2011.03.022 [PubMed]

10. Lei C, Har YC, Abdullah KL. Informational needs of breast cancer patients on chemotherapy: Differences between patients' and nurses' perceptions. Asian Pac J Cancer Prev. 2011; 12(3): 797-802. PMID: 21627386 [PubMed]

11. Husson O, Mols F, Van de Poll-Franse L. The relation between information provision and health-related quality of life, anxiety and depression among cancer survivors: A systematic review. Ann Oncol. 2011; 22(4): 761-72. PMID: 20870912 DOI: 10.1093/annonc/mdq413 [PubMed]

12. Lee YM, Francis K, Walker J, Lee SM. What are the information needs of Chinese breast cancer patients receiving chemotherapy? Eur J Oncol Nurs. 2004; 8(3): 224-33. PMID: 15304230 DOI: 10.1016/j.ejon.2003.12.006 [PubMed]

13. Kent EE, Arora NK, Rowland JH, Bellizzi KM, Forsythe LP, Hamilton AS, et al. Health information needs and health-related quality of life in a diverse population of long-term cancer survivors. Patient Educ Couns.
2012; 89(2): 345-52. PMID: 23021856 DOI: 10.1016/j.pec.2012.08.014 [PubMed]

14. Kuijpers W, Groen WG, Loos R, Oldenburg HS, Wouters MW, Aaronson NK, et al. An interactive portal to empower cancer survivors: A qualitative study on user expectations. Support Care Cancer. 2015; 23(9): 2535-42. PMID: 25876157 DOI: 10.1007/s00520-015-2605-0 [PubMed]

15. Fourie I. Promoting awareness of information needs and realising gaps in knowledge: Examples from healthcare. Bladen voor documentatie. 2013; 3: 3240 .

16. Kimiafar K, Sarbaz M, Shahid Sales S, Esmaeili M, Javame Ghazvini Z. Breast cancer patients' information needs and information-seeking behavior in a developing country. Breast J. 2016; 28: 156-60. PMID: 27318171 DOI: 10.1016/j.breast.2016.05.011 [PubMed]

17. Maddock C, Lewis I, Ahmad K, Sullivan R. Online information needs of cancer patients and their organizations. Ecancermedicalscience. 2011; 5: 235. PMID: 22276067 DOI: 10.3332/ecancer.2011.235 [PubMed]

18. Wang SY, Kelly G, Gross C, Killelea BK, Mougalian S, Presley C, et al. Information needs of older women with early-stage breast cancer when making radiation therapy decisions. Int J Radiat Oncol Biol Phys. 2017; 98(4): 733-40. PMID: 28366581 DOI: 10.1016/j.ijrobp.2017.02.001 [ubMed]

19. Burton M, Collins KA, Lifford KJ, Brain K, Wyld L, Caldon $\mathrm{L}$, et al. The information and decision support needs of older women (> $75 \mathrm{yrs}$ ) facing treatment choices for breast cancer: A qualitative study. Psychooncology. 2015; 24(8): 878-84. PMID: 25534045 DOI: 10.1002/pon.3735 [PubMed]

20. Li W, So WK, Fong DY, Lui LY, Lo JC, Lau SF. The information needs of breast cancer patients in Hong Kong and their levels of satisfaction with the provision of information. Cancer Nurs. 2011; 34(1): 49-57. PMID: 21139454 DOI: 10.1097/NCC.0b013e3181ef77a0 [PubMed]

21. Nader EA, Kourie HR, Ghosn M, Karak FE, Kattan J, Chahine G, et al. Informational needs of women with breast cancer treated with chemotherapy. Asian Pac J Cancer Prev. 2016; 17(4): 1797-800. PMID: 27221855 DOI: 10.7314/apjcp.2016.17.4.1797 [PubMed]

22. Findik UY. The Information needs of women who have undergone breast cancer surgery in the west of Turkey. J Cancer Educ. 2015; 32(3): 432-7. PMID: $26691514 \quad$ DOI: $\quad 10.1007 / \mathrm{s} 13187-015-0965-2$ [PubMed]

23. Halbach SM, Ernstmann N, Kowalski C, Pfaff $\mathrm{H}$, Pförtner TK, Wesselmann S, et al. Unmet information needs and limited health literacy in newly diagnosed breast cancer patients over the course of cancer treatment. Patient Educ Couns. 2016; 99(9): 1511-8. PMID: 27378079 DOI: 10.1016/j.pec.2016.06.028 [PubMed]

24. Girgis A, Stacey F, Lee T, Black D, Kilbreath S. 
Priorities for women with lymphoedema after treatment for breast cancer: Population based cohort study. BMJ, 2011. 342: d3442. PMID: 21693532 DOI: 10.1136/bmj.d3442 [PubMed]

25. Douma KF, Koning CC, Zandbelt LC, de Haes HC, Smets EM. Do patients' information needs decrease over the course of radiotherapy? Support Care Cancer. 2012; 20(9): 2167-76. PMID: 22081119 DOI: 10.1007/s00520-011-1328-0 [PubMed]

26. Feldman-Stewart D, Madarnas $\mathrm{Y}$, Mates M, Tong C, Grunfeld E, Verma S, et al. Information needs of postmenopausal women with hormone receptor positive early-stage breast cancer considering adjuvant endocrine therapy. Patient Educ Couns. 2013; 93(1): 114-21. PMID: 23747087 DOI: 10.1016/j.pec.2013.03.019 [PubMed]

27. Yeşilyurt DS, Findık ÜY. Informational needs of postmastectomy patients. J Breast Health. 2016; 12(4): 155-7. PMID: 28331754 DOI: 10.5152/tjbh.2016.3062 [PubMed]

28. Schmidt A, Ernstmann N, Wesselmann S, Pfaff $\mathrm{H}$, Wirtz M, Kowalski C. After initial treatment for primary breast cancer: Information needs, health literacy, and the role of health care workers. Support Care Cancer. 2016; 24(2): 563-71. PMID: 26108171 DOI: $10.1007 / \mathrm{s} 00520-015-2814-6$ [PubMed]

29. Kowalski C, Lee SY, Ansmann L, Wesselmann S, Pfaff H. Meeting patients' health information needs in breast cancer center hospitals-a multilevel analysis. BMC Health Serv Res. 2014; 14(1): 601. PMID: 25422099 DOI: $10.1186 / \mathrm{s} 12913-014-0601-6$ [PubMed]

30. Almyroudi A, Degner LF, Paika V, Pavlidis N, Hyphantis T. Decision making preferences and information needs among Greek breast cancer patients. Psychooncology. 2011; 20(8): 871-9. PMID: 20623805 DOI: 10.1002/pon.1798 [PubMed]

31. Halkett GK, Kristjanson LJ, Lobb E, Little J, Shaw T, Taylor M, et al. Information needs and preferences of women as they proceed through radiotherapy for breast cancer. Patient Educ Couns. 2012; 86(3): 396404. PMID: 21664788 DOI: 10.1016/j.pec.2011.05.010 [PubMed]

32. Schmidt A, Kowalski C, Pfaff $H$, Wesselmann S, Wirtz M, Ernstmann N. The influence of health literacy on information needs among women newly diagnosed with breast cancer, with special reference to employment status. J Health Commun. 2015; 20(10): 1177-84. PMID: 26086892 DOI: 10.1080/10810730.2015.1018626 [PubMed]

33. Albada A, Ausems MG, Otten R, Bensing JM, van Dulmen $S$. Use and evaluation of an individually tailored website for counselees prior to breast cancer genetic counseling. J Cancer Educ. 2011; 26(4): 67081. PMID: 21533850 DOI: $10.1007 / \mathrm{s} 13187-011-$ 0227-x [PubMed]

34. Obeidat R, Khrais HI. Information needs and disclosure preferences among Jordanian women diagnosed with breast cancer. J Cancer Educ. 2015; 30(1): 94-9. PMID: 24781932 DOI: 10.1007/s13187014-0665-3 [PubMed]
35. White JA, Matthews E, Wallace L, Poormon L. Informational needs of breast cancer patients related to lymphedema. Rehabilitation Oncology. 2015; 33(1): 58-63.

36. Bei AW, Lai MT, Choi KC, Li PW, So WK. Factors in the prioritization of information needs among Hong Kong Chinese breast cancer patients. Asia Pac J Oncol Nurs. 2015; 2(3): 176-85. PMID: 27981112 DOI: 10.4103/2347-5625.163620 [ubMed]

37. Ussher JM, Perz J, Gilbert E. Information needs associated with changes to sexual wellbeing after breast cancer. J Adv Nurs. 2013; 69(2): 327-37. PMID: 22500731 DOI: $10.1111 /$ j.13652648.2012.06010.x [PubMed]

38. Goss C, Deledda G, Bottacini A, Chiodera F, Mazzi MA, Ballarin $\mathrm{M}$, et al. Information needs of female Italian breast cancer patients during their first oncological consultation. Eur J Oncolo Nurs. 2015; 19(5): 451-7. PMID: 25788023 DOI: 10.1016/j.ejon.2015.02.003 [PubMed]

39. Tan AS, Nagler RH, Hornik RC, DeMichele A. Evolving information needs among colon, breast, and prostate cancer survivors: Results from a longitudinal mixedeffects analysis. Cancer Epidemiol Biomarkers Prev. 2015; 24(7): 1071-8. PMID: 25979968 DOI: 10.1158/1055-9965.EPI-15-0041 [PubMed]

40. Bennett MI, Flemming $\mathrm{K}$, Closs SJ. Education in cancer pain management. Curr Opin Support Palliat Care. 2011; 5(1): 20-4. PMID: 21157351 DOI: 10.1097/SPC.0b013e328342c607 [ubMed]

41. Kwok C, White K. Perceived information needs and social support of Chinese-Australian breast cancer survivors. Support Care Cancer. 2014; 22(10): 26519. PMID: 24752565 DOI: 10.1007/s00520-014-2252$\mathrm{x}$ [PubMed]

42. Faller H, Brähler E, Härter $M$, Keller M, Schulz $H$, Wegscheider $\mathrm{K}$, et al. Unmet needs for information and psychosocial support in relation to quality of life and emotional distress: A comparison between gynecological and breast cancer patients. Patient Educ Couns. 2017; 100(10): 1934-42. PMID: $28592366 \quad$ DOI: $10.1016 /$ j.pec.2017.05.031 [PubMed]

43. Cardoso F, Harbeck N, Mertz S, Fenech D. Evolving psychosocial, emotional, functional, and support needs of women with advanced breast cancer: Results from the Count Us, Know Us, Join Us and Here \& Now surveys. Breast. 2016; 28: 5-12. PMID: 27161410 DOI: $10.1016 /$ j.breast.2016.04.004 [PubMed]

44. Poon PCM. The information needs, perceptions of communication and decision-making process of cancer patients receiving palliative chemotherapy. Journal of Pain Management. 2012; 5(1): 93-105.

45. de Bock G, Bonnema J, Zwaan RE, van de Velde CJ, Kievit J, Stiggelbout AM. Patient's needs and preferences in routine follow-up after treatment for breast cancer. Br J Cancer. 2004; 90(6): 1144-50. PMID: 15026793 DOI: 10.1038/sj.bjc.6601655 [PubMed] 
46. Adams E, McCann L, Armes J, Richardson A, Stark D, Watson E, et al. The experiences, needs and concerns of younger women with breast cancer: a meta-ethnography. Psychooncology. 2011; 20(8): 851-61. PMID: 20597066 DOI: 10.1002/pon.1792 [PubMed]

47. Recio-Saucedo A, Gerty S, Foster C, Eccles D, Cutress RI. Information requirements of young women with breast cancer treated with mastectomy or breast conserving surgery: A systematic review. Breast. 2016. 25: 1-13. PMID: 26801410 DOI: 10.1016/j.breast.2015.11.001 [PubMed]

48. Valero-Aguilera B, Bermúdez-Tamayo C, GarcíaGutiérrez JF, Jiménez-Pernett J, Cózar-Olmo JM, Guerrero-Tejada $\mathrm{R}$, et al. Information needs and Internet use in urological and breast cancer patients. Supportive Care in Cancer. 2014; 22(2): 545-52. PMID: 24122406 DOI: 10.1007/s00520-013-2009-y [PubMed]

49. Remmers H, Holtgräwe M, Pinkert C. Stress and nursing care needs of women with breast cancer during primary treatment: A qualitative study. Eur J Oncol Nurs. 2010; 14(1): 11-6. PMID: 19748314 DOI: 10.1016/j.ejon.2009.07.002 [PubMed]

50. Fu MR, Xu B, Liu Y, Haber J. 'Making the best of it': Chinese women's experiences of adjusting to breast cancer diagnosis and treatment. J Adv Nurs. 2008; 63(2): 155-65. PMID: 18537844 DOI: 10.1111/j.1365-2648.2008.04647.x [PubMed]

51. Balneaves LG, Panagiotoglou D, Brazier AS, Lambert LK, Porcino A, Forbes M, et al. Qualitative assessment of information and decision support needs for managing menopausal symptoms after breast cancer. Support Care Cancer. 2016; 24(11): 4567-75. PMID: $27278271 \quad$ DOI: $\quad 10.1007 / \mathrm{s} 00520-016-3296-\mathrm{x}$ [PubMed]

52. Miyashita M, Ohno S, Kataoka A, Tokunaga E, Masuda $\mathrm{N}$, Shien $\mathrm{T}$, et al. Unmet information needs and quality of life in young breast cancer survivors in Japan. Cancer Nurs. 2015; 38(6): E1-E11. PMID: 25254410 DOI: $10.1097 /$ NCC.0000000000000201 [PubMed]

53. Tautz E, Momm F, Hasenburg A, Guethlin C. Use of complementary and alternative medicine in breast cancer patients and their experiences: A crosssectional study. Eur J Cancer. 2012; 48(17): 3133-9. PMID: 22633625 DOI: 10.1016/j.ejca.2012.04.021 [PubMed]

54. Hodgkinson K, Butow P, Hobbs KM, Wain G. After cancer: the unmet supportive care needs of survivors and their partners. J Psychosoc Oncol. 2007; 25(4): 89-104. $\quad$ PMID: 18032267 DOI: 10.1300/J077v25n04_06 [PubMed]

55. Hyodo I, Amano N, Eguchi K, Narabayashi M, Imanishi J, Hirai $M$, et al. Nationwide survey on complementary and alternative medicine in cancer patients in Japan. J Clin Oncol. 2005; 23(12): 264554. PMID: 15728227 DOI: 10.1200/JCO.2005.04.126 [PubMed]

56. Huebner J, Follmann M. Complementary medicine in guidelines of the German guideline program in oncology: Comparison of the evidence base between complementary and conventional therapy. J Cancer Res Clin Oncol. 2013; 139(9): 1481-8. PMID: 23817695 DOI: $10.1007 / \mathrm{s} 00432-013-1464-7$ [PubMed]

57. Tung HH, Lin CY, Chen KY, Chang CJ, Lin YP, Chou CH. Selfmanagement intervention to improve self-care and quality of life in heart failure patients. Congest Heart Fail. 2013; 19(4): E9-E16. PMID: 23279120 DOI: $10.1111 / \operatorname{chf} .12014$ [PubMed]

58. Bandura A. Self-efficacy: Toward a unifying theory of behavioral change. Psychol Rev. 1977; 84(2): 191215. PMID: 847061 DOI: $10.1037 / / 0033-$ 295x.84.2.191 [PubMed]

59. Chiaranai C, Salyer J, Best A. Self-care and quality of life in patients with heart failure. Thai J Nurs Res. 2009; 13(4): 302-17.

60. Williams SA, Schreier AM. The effect of education in managing side effects in women receiving chemotherapy for treatment of breast cancer. Oncol Nurs Forum. 2004; 31(1): E16-23. PMID: 14722602 DOI: 10.1188/04.ONF.E16-E23 [PubMed]

61. Partridge AH. Cancer survivorship and the young breast cancer patient: addressing the important issues. Oncologist. 2013; 18(8): e19-20. PMID: 23986342 DOI: 10.1634/theoncologist.2013-0300 [PubMed]

62. Koopman C, Butler LD, Classen C, Giese-Davis J, Morrow GR, Westendorf J, et al. Traumatic stress symptoms among women with recently diagnosed primary breast cancer. J Trauma Stress. 2002; 15(4): 277-87. PMID: 12224799 DOI: 10.1023/A:1016295610660 [PubMed]

63. Matchim Y, Armer JM, Stewart BR. Mindfulnessbased stress reduction among breast cancer survivors: A literature review and discussion. Oncol Nurs Forum. 2011; 38(2): E61-71. PMID: 21356643 DOI: 10.1188/11.ONF.E61-E71 [PubMed]

64. Günüșen N, İnan FŞ, Üstün B. Experiences of Turkish women with breast cancer during the treatment process and facilitating coping factors. Asian Pac J Cancer Prev. 2013; 14(5): 3143-9. PMID: 23803093 [PubMed]

65. Henderson PD, Gore SV, Davis BL, Condon EH. African American women coping with breast cancer: A qualitative analysis. Oncol Nurs Forum. 2003; 30(4): 641-7. PMID: 12861324 DOI: 10.1188/03.0NF.641-647 [PubMed]

66. Crowley SA, Foley SM, Wittmann D, Jagielski $\mathrm{CH}$, Dunn RL, Clark PM, et al. Sexual health concerns among cancer survivors: testing a novel informationneed measure among breast and prostate cancer patients. PMID: 26076657 DOI: 10.1007/s13187015-0865-5 [PubMed]

67. Pujols $\mathrm{Y}$, Meston CM, Seal BN. The association between sexual satisfaction and body image in women. J Sex Med. 2010; 7(2): 905-16. PMID: 19968771 DOI: 10.1111/j.1743-6109.2009.01604.x [PubMed]

68. Speer JJ, Hillenberg B, Sugrue DP, Blacker C, Kresge 
CL, Decker VB, et al. Study of sexual functioning determinants in breast cancer survivors. Breast J. 2005; 11(6): 440-7. PMID: 16297089 DOI: 10.1111/j.1075-122X.2005.00131.x [PubMed]

69. Burwell SR, Case LD, Kaelin C, Avis NE. Sexual problems in younger women after breast cancer surgery. J Clin Oncol. 2006; 24(18): 2815-21. PMID: 16782919 DOI: $10.1200 / J C 0.2005 .04 .2499$ [PubMed]

70. Schultz WC, Van de Wiel HB. Sexuality, intimacy, and gynecological cancer. J Sex Marital Ther. 2003; 29(Suppl 1): 121-8. PMID: 12735096 DOI: $10.1080 / 713847128$ [PubMed]

71. Lemieux L, Kaiser S, Pereira J, Meadows LM. Sexuality in palliative care: Patient perspectives. Palliat Med. 2004; 18(7): 630-7. PMID: 15540672 DOI: 10.1191/0269216304pm941oa [PubMed]

72. Stabile C, Goldfarb S, Baser RE, Goldfrank DJ, AbuRustum NR, Barakat RR, et al. Sexual health needs and educational intervention preferences for women with cancer. Breast Cancer Res Treat. 2017; 165(1): 77-84. PMID: 28547655 DOI: 10.1007/s10549-0174305-6 [PubMed]
73. Gopal RL, Beaver $\mathrm{K}$, Barnett $\mathrm{T}$, Ismail NS. A comparison of the information needs of women newly diagnosed with breast cancer in Malaysia and the United Kingdom. Cancer Nurs. 2005 MarApr;28(2):132-40. PMID: 15815183 DOI: 10.1097/00002820-200503000-00007 [PubMed]

74. Takahashi M, Ohno S, Inoue H, Kataoka A, Yamaguchi $\mathrm{H}$, Uchida $\mathrm{Y}$, et al. Impact of breast cancer diagnosis and treatment on women's sexuality: A survey of Japanese patients. Psychooncology. 2008; 17(9): 901-7. PMID: 18074406 DOI: 10.1002/pon.1297 [PubMed]

75. Gould J, Grassau P, Manthorne J, Gray RE, Fitch MI. 'Nothing fit me': Nationwide consultations with young women with breast cancer. Health Expect. 2006; 9(2): 158-73. PMID: 16677195 DOI: 10.1111/j.1369-7625.2006.00383.x [리리]

76. Zeng YC, Liu X, Loke AY, Addressing sexuality issues of women with gynaecological cancer: Chinese nurses' attitudes and practice. J Adv Nurs. 2012; 68(2): 280-92. PMID: 21658098 DOI: 10.1111/j.1365-2648.2011.05732.x [PubMed] 